\title{
ON THE CHANGE IN LENGTH OF SOFT IRON IN AN ALTERNATING MAGNETIC FIELD.
}

By Louis W. Austin.

$\mathrm{W}$

HILE doing some work with one of my students on the change of length of iron due to magnetism, it occurred to me that there must be a similar effect in an alternating field, but perhaps modified in amount or character by the more or less rapid alternations. If the change in length requires an appreciable time for its completion, such modifications, it would seem must exist, and the probability of this seemed to me great enough to make it worth while to investigate the subject experimentally.

The instrument used in the investigation is based on an old principle long used in the lecture room to show qualitatively the expansion of solids due to heat. In its original form it consisted of a small roller placed under one end of a bar, which was held fast at the other end. When the bar was heated it expanded the roller rolled and by the movement of a pointer or small mirror attached to it showed to the class the expansion of the bar. In the improved form the horizontal roller is replaced by a vertical hanging fiber or fine wire which is held between vertical plates of plate glass about $2 \mathrm{~cm}$. square, so that it is rolled when one of the plates is moved just as one might roll a thread between the thumb and finger. Below the plates a larger glass fiber of sufficient rigidity is attached to the rolling fiber and this bears a mirror and water damper. A second rolling fiber is hung between the plates at a little distance from the first in order to keep the surfaces apart to prevent friction. In the first form of instrument made and the one used in this work the fixed plate was cemented to a block of wood and the movable plate against which the iron rested was pressed against it by means of a rubber band. With this arrangement $I$ have been able to get results with a glass fiber $0.0 \mathrm{I} \mathrm{mm}$. in diameter, but in general fibers 
as small as this are extremely difficult to adjust and are very apt to break between glass surfaces. I am now experimenting with other surfaces in the hope of getting better friction contact and less trouble in adjustment. I hope soon to be able to communicate a paper on this work.

Most of the observations recorded in the present paper were taken with a glass fiber about $0.06 \mathrm{~mm}$. in diameter, though for a few of the later ones a fine wire was used. The latter observations being reduced to the scale of the former on the supposition that the maximum elongation with direct current was the same in both cases. Each of the observations given in the tables is the mean of from six to twelve observed throws and the good behavior of the instrument may be judged from the fact that in none of the series used, except in the case of frequencies greater than sixty per sec., did any of the single observations differ by more than two per cent. from the mean. The absolute calibration was made rather roughly by noting the distance the cross hair of the telescope appeared to move on a scale half a meter away when the moving plate was pushed forward by a fine micrometer screw.

The preliminary observations on the change of length in an alternating field were made on a soft iron bar $26 \mathrm{~cm}$. long and 0.7 $\mathrm{cm}$. in diameter, the same one with which I had been working with a direct field. The source of current was the city incandescent lighting circuit having a frequency of $\mathrm{I} 20$ per sec. by day and 60 per sec. by night. I found that the curves showing the relation between change of length and strength of field were similar in form for the alternating and direct currents, but that the maximum in the case of the alternating field fell some ten or fifteen per cent. below that produced by direct field, and that this decrease was greater with the frequency 120 than with 60 per sec.

These experiments were rendered difficult by the large heat expansion produced by the Foucault currents and hysteresis which of course made accurate observation somewhat uncertain. I have thus far found no remedy for the latter difficulty, but have rid myself of the former for the most part by replacing the solid iron bar by a bundle of wires of soft Swedish iron firmly bound together and insulated with varnish. The observations given in this paper 
were taken with such a bundle consisting of ten wires $27.5 \mathrm{~cm}$. long and $\mathrm{I} .4 \mathrm{~mm}$. in diameter. The magnetizing solenoid was 24 $\mathrm{cm}$. long with $\mathrm{I} 2$ turns per $\mathrm{cm}$. The dilatometer with the fiber used (about $0.06 \mathrm{~mm}$.) gave a magnification of about 127000 times with the scale $360 \mathrm{~cm}$. distant. This gave a maximum reading of I $4.5 \mathrm{~cm}$. on the scale with direct field.

With apparatus kindly placed at my disposal by the department of electrical engineering ${ }^{1} \mathrm{I}$ was able to work with currents varying in frequency from Io to I 3 I per sec. The values of $H$ varied from 7.5 to 375 , approximately, no correction being made for the influence of the ends of the solenoid. The larger value was a little more than enough to bring the iron back to its original length. With the stronger fields, especially with the higher frequencies, the heat due to hysteresis rendered the observations somewhat uncertain. I have been able, however, within the limits of field and frequency used, to get very consistent results by taking the readings by breaking the circuit and noting the distance the cross hair appeared to spring back on the scale. This motion is so sudden and so dead-beat that I do not think any larger error is introduced by the heat drift. Thinking that the water damper might influence readings taken in this way, several observations were taken without it, but no change in the throws could be detected.

In Table. I are given the changes in length expressed in ten-mil-

TABLE I.

\begin{tabular}{r|c|c|c|c|c}
\hline \multicolumn{1}{c|}{$H$} & $\begin{array}{c}\text { Direct } \\
\text { Current. }\end{array}$ & $\begin{array}{c}\text { 1o } \\
\text { per sec. }\end{array}$ & $\begin{array}{c}30 \\
\text { per sec. }\end{array}$ & $\begin{array}{c}60 \\
\text { per sec. }\end{array}$ & $\begin{array}{c}\text { x20 } \\
\text { per sec. }\end{array}$ \\
\hline 7.5 & 1.7 & 2.2 & 2.2 & & \\
15.0 & 9.0 & 13.2 & 13.2 & 14.4 & 13.2 \\
30.0 & 29.5 & 32.4 & 29.0 & 25.8 & 24.5 \\
45.0 & 38.5 & 34.5 & 35.0 & 31.0 & 29.4 \\
75.0 & 41.5 & 39.8 & 36.6 & 34.1 & 31.0 \\
150.0 & 30.1 & 28.0 & & 27.0 & \\
225.0 & 20.2 & 18.3 & & & \\
300.0 & & 8.0 & & & \\
375.0 & -2.8 & $-4.0 ?$ & & & \\
\hline \hline
\end{tabular}

1 My special thanks are due to Mr. M. C. Beebe, instructor in electrical engineering, for his kindness in managing the electrical machinery used. 
lionths of the length for direct current and for currents with alternations of $10,30,60$ and 120 per sec., the strength of field varying from 7.5 to $375 \mathrm{C}$. G. S. The maximum comes at a value of $H$ of about 75 in all the series, and the general course of the phenomenon is similar in all. With the lower values of $H$ the elongations seem to increase more rapidly with the alternating currents than with the direct. This is perhaps due to the fact that the currents were read with an ammeter which indicated the square root of the mean square of current strength, which is very probably less than the effective strength of the alternating currents in this

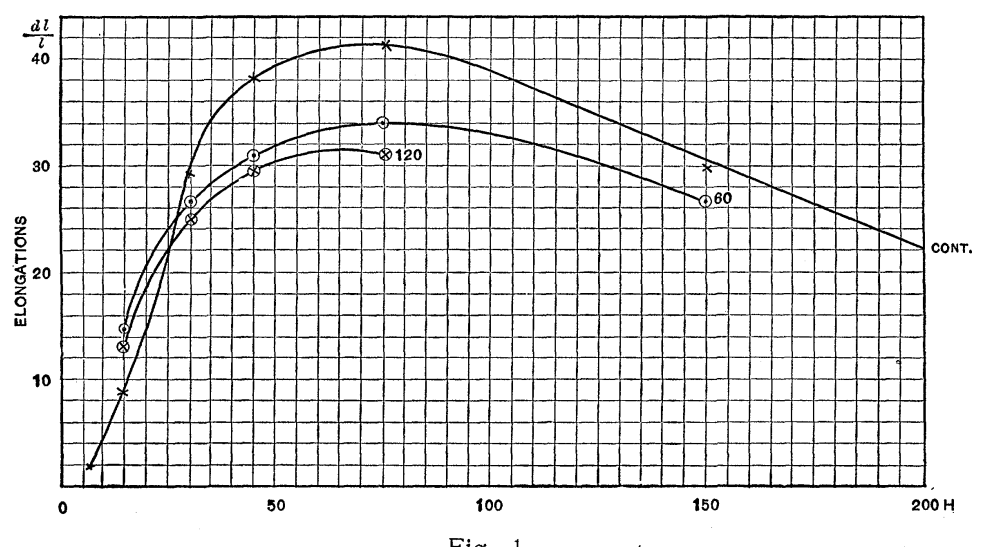

Fig. 1.

phenomenon. With all values of $H$ above ${ }_{5} 5$ the alternating current elongations fall below those with direct current. The curves of Fig. I show these relations graphically for direct current and frequencies of 60 and 120 .

TABLE II.

\begin{tabular}{c|c||c|c}
\hline Frequencies. & Maxim. expansion. & Frequencies. & Maxim. expansion. \\
\hline Direct current & 41.5 & 66 per sec. & 33.2 \\
10 per sec. & 39.8 & 81 & 32.2 \\
16 & 39.6 & 99 & 31.5 \\
30 & 36.6 & 120 & 31.0 \\
40 & 35.2 & 131 & 30.7 \\
60 & 34.0 & & \\
\hline
\end{tabular}


Table II. gives the maximum elongation corresponding to different frequencies. A steady drop is noted as the frequencies become higher, which becomes less marked toward the end. Fig. 2, with its rather flat approximately exponential curve, shows the character of the change much better than the table can do.

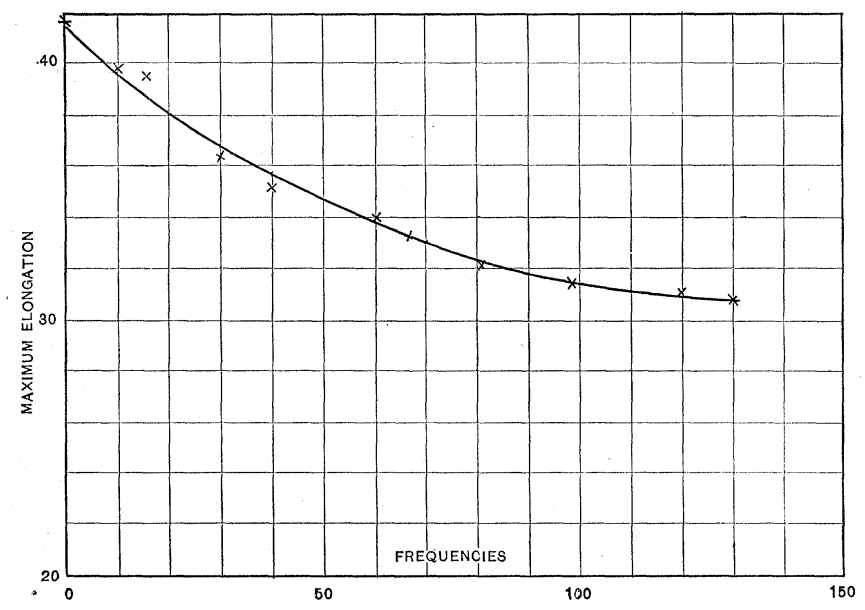

Fig. 2.

An attempt was made at one time to use the secondary currents of a transformer having a Wehnelt interrupter in the primary. The frequency as estimated from the pitch was about 800 per sec. The heating effects were so great, however, that no readings of value could be taken, the scale going by the eye far too rapidly to be read. The maximum certainly fell much below any of the values given in the table and was estimated to be about half that found with the frequency of I 3 I. The results of the investigation may be briefly stated as follows :

I. The general course of the change in length of soft iron in an alternating magnetic field is the same as in the direct field.

2. For all values of $H$ above 25 , and at least as high as 375 , the length of the soft-iron specimen is less with an alternating than with a direct field.

3. The maximum elongation gradually becomes less as the frequency of the alternations is increased. 
This continued decrease of the maximum elongation with increasing frequency is perhaps the most interesting fact brought out. It appears as though there were an appreciable viscosity ${ }^{1}$ in the molecular movements depending on the rapidity of the alterations. The last two results may, it seems to me, have a connection with the observations of several physicists ${ }^{2}$ on the decrease of permeability of iron when subjected to rapid magnetic reversals. The recent work of Niethammer ${ }^{3}$ and of Wien ${ }^{4}$ on this subject also shows a correspondence with the third result, Niethammer finding a percentage decrease in permeability with increasing frequency, agreeing fairly closely numerically with the results in this paper. Wien's percentage of decrease is considerably smaller. It can hardly be said, however, that the decrease in the maximum expansion is due merely to decreased induction, since if this were so it would seem that an increase of the field should raise the induction so as to produce the same maximum elongation as before, the only result being to make the maximum correspond to a slightly greater value of $H$. Since this is not the case the decreased maximum elongation cannot be considered a direct result of the decreased permeability, but their quantitatively similar decrease would indicate that they both may follow from the same molecular causes.

Exactly what course the elongation curves take with higher values of $H$, I have not been able to discover on account of the heating disturbances. The observations merely indicate that with the alternating current the iron is brought back to its initial length at a lower

${ }^{1}$ Since the completion of this work I have come across the observations of A. M. Mayer (Phil. Mag., I873, Vol. 46, p. I77) on the time required for the full magnetic expansion to take place. These are the only observations made up to this time, so far as I am aware, that bear on the time factor in this phenomenon. He found that with his strongest current ( 25 Bunsen cells) $\frac{1}{10}$ second was required for the expansion and ${ }_{10}^{3}$ second for the corresponding retraction. While these estimates are certainly rough, they indicate the presence of the same appreciable viscosity which shows itself in the alternating field.

2 Warburg and Hönig, Wied. Ann., Vol. 20, r883, p. $8 \mathbf{r}_{4}$.

Tanakadate, Phil. Mag., Vol. 28, I889, p. 207.

Klemencic, Sitzungsber. d. k. Gesellsch. d. Wissench. zu Wien, Vol. 103, p. I7, I894.

Weihe, Wied. Ann., Vol. 61, I897, p. 578 .

3 Niethammer, Wied. Ann., Vol. 66, 1898, p. 29.

${ }^{4}$ M. Wien, Wied. Ann., Vol. 66, I898, p. 859. 
value of $H$ than with the continuous current. Of the course of the phenomenon beyond this point I can say nothing, but I hope in the near future to overcome the difficulties which have restricted the scope of the work and to investigate the effects both of stronger fields and higher frequencies.

Physical Laboratory of the University of Wisconsin, January io, igoo. 